\title{
High-Quality Model Generation for Finite Element Simulation of Tissue Deformation
}

\author{
Orcun Goksel and Septimiu E. Salcudean \\ Department of Electrical and Computer Engineering \\ University of British Columbia, Vancouver, Canada \\ \{orcung, tims\}@ece.ubc.ca
}

\begin{abstract}
In finite element simulation, size, shape, and placement of the elements in a model are significant factors that affect the interpolation and numerical errors of a solution. In medical simulations, such models are desired to have higher accuracy near features such as anatomical boundaries (surfaces) and they are often required to have element faces lying along these surfaces. Conventional modelling schemes consist of a segmentation step delineating the anatomy followed by a meshing step generating elements conforming to this segmentation. In this paper, a one-step energy-based model generation technique is proposed. An objective function is minimized when each element of a mesh covers similar image intensities while, at the same time, having desirable FEM characteristics. Such a mesh becomes essential for accurate models for deformation simulation, especially when the image intensities represent a mechanical feature of the tissue such as the elastic modulus. The use of the proposed mesh optimization is demonstrated on synthetic phantoms, 2D/3D brain MR images, and prostate ultrasound-elastography data.
\end{abstract}

\section{Introduction}

The finite element method (FEM) is a common technique for medical simulations. Its speed and accuracy depend on the number of nodes/elements used and their shape and placement in the domain. In this paper, a single-step and fullyautomatic variational modelling approach is presented to produce good FEM meshes for given tissue domains in both 2D and 3D. The method, which aligns FEM elements to group similar intensities while still keeping element shapes relatively good for FEM, is applicable on most medical imaging modalities. Its use becomes particularly important if the input image represents a mechanical feature distribution of the tissue such as Young's modulus.

In the conventional modelling methods for tissue FEM simulation, a discrete representation of the anatomy of interest is obtained from an intensity image/volume by employing two steps, segmentation and meshing. Segmentation, which consists of recognition and delineation of anatomy, has been studied in several medical contexts using numerous different approaches. Although automatic segmentation techniques do exist, recognition is not a computationally well-defined problem and thus is usually achieved with manual intervention leading to semi-automatic implementations. In contrast, delineation, which in many 
cases can be stated roughly as grouping similar pixels, allows for algorithmic approaches. Nonetheless, segmentation overall often requires some a priori information about both the anatomy and the medical imaging modality.

The result of segmentation is a representation of the organ boundary, which is often in an explicit form such as a surface mesh, although implicit representations are also viable. This anatomical boundary is then supplied to a meshing scheme, which tiles the space with elements while ensuring some geometrical measures of such elements meet given or well-known criteria. Popular meshing schemes include octree-based methods, Delaunay approaches, and advancing fronts, most of which were developed and tuned for modelling of mechanical structures and systems [1. The final mesh is then used for simulating tissue deformation for procedures such as laparoscopic surgery, brain surgery, and brachytherapy.

Most segmentation methods require some sort of a manual intervention not only demanding the scarcely available time of health professionals but also preventing an automatic modelling for FEM. The surface mesh generated during the segmentation is often left unchanged by the meshing software since this surface is used as a boundary constraint for meshing. Thus, for a meshing scheme to guarantee good FEM elements using a limited number of nodes/elements, these surfaces should either be inherently FEM-friendly delineations or should be fixed using some intermediate steps. Furthermore, boundary conforming meshes, when the inside and outside of an anatomical feature are to be discretized with no element faces cutting the boundary, present a challenge for otherwise successful schemes such as Delaunay tessellation [1. The technique presented in this paper intrinsically overcomes these issues producing high-quality FEM elements that are also aligned with the features in a given image.

\section{Methods}

\subsection{Element Shape Optimization}

Let $u(x)$ be the deformation of a point $x$ within a continuous domain $\mathcal{M} \subset \mathbb{R}^{n}$, where this deformation is approximated using the FEM by a piece-wise linear discrete function $g_{\mathcal{T}}(x)$ over a tessellation $\mathcal{T}$. During such modelling of deformation, the two main sources of error are the interpolation errors for the approximation to the function and its gradient (which is strain for deformation), and the numerical errors during the solution of the approximation [2].

Variational (energy-minimization based) methods were shown to produce successful meshes for various applications including meshes suitable for FEM [1]. Recent studies showed that a primal mesh energy definition is superior to the Voronoi-based approaches 3 . It was also shown that the following error [1]:

$$
E_{G}=\left\|u(\mathbf{x})-g_{\mathcal{T}}(\mathbf{x})\right\|=\frac{1}{n+1} \sum_{i} \mathbf{x}_{i}^{2}\left|\Omega_{i}\right|-\int_{\mathcal{M}} \mathbf{x}^{2} d \mathbf{x}
$$

is minimized by the following two operations: a Delaunay tessellation of given node locations, and a relocation of a node $\mathbf{x}_{i}$ to the weighted average of the 


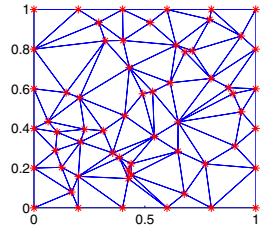

(a)

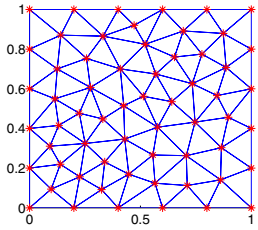

(b)

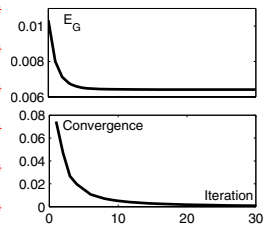

(c)

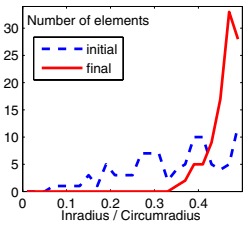

(d)

Fig. 1. A random mesh initialization (a), its optimized configuration (b), the energy and convergence during this optimization (c), and the initial/final $\rho$ distributions (d)

element circumcenters in its 1-ring neighbourhood $\Omega_{i}$, where the weighting is the element volume. Consequently, a (local) minimizer mesh for $E_{G}$ is found using Lloyd's relaxation by alternately re-tessellating and relocating the nodes 1 . Figure 1(a-b) shows an initial mesh of randomly-distributed 61 nodes and its optimized configuration after having converged. The energy $E_{G}$ and the nodal position convergence $\left\|\mathbf{x}_{i}^{t+1}-\mathbf{x}_{i}^{t}\right\| /\left\|\mathbf{x}_{i}^{t}\right\|$ at iteration $t$ are presented in Fig.1 (c).

Comparing two meshes using merely a single error measure is an attractive approach, not only simply to choose the better mesh, but also to formulate energy definitions that can derive variational schemes. No such single measure has been developed in the literature. For predicting the worst interpolationerror in a single element, various measures were proposed in [2]. The inradiusto-circumradius ratio $\rho$, which was presented in [1] as the fairest comparison in $3 \mathrm{D}$ that punishes all types of poor-geometry elements including slivers, is used in this paper. This ratio is maximized at $1 / 2$ for the equilateral triangle and at $1 / 3$ for the regular tetrahedron. Similarly to [1, normalized histograms of this ratio are used to compare meshes, where a desired mesh has a histogram more compacted towards higher ratios as displayed in Fig.1(d) for the initial and optimized meshes presented above. Since a qualitative comparison of curves can be difficult, we propose the following measures in this paper: $(i)$ mean quality error mean $\left(\frac{1}{n}-\rho\right),($ ii $)$ harmonic-mean quality error $1 / \sum \frac{1}{\frac{1}{n}-\rho},($ iii $) \mathcal{L}_{2}$-norm quality error $\left|\frac{1}{n}-\rho\right|^{2}$, and $(i v)$ worst quality error $\max \left(\frac{1}{n}-\rho\right)$. All these quality errors improve during the sample optimization above as seen in Table 1

Table 1. The inradius-to-circumradius ratios for each row corresponding to the meshes presented in Figs.11(a,b), Figs.2(a,c,d), and Figs. 3](c,d), respectively

\begin{tabular}{|l|c|c|c|c|}
\hline \multicolumn{1}{|c|}{ Error measures based on $\rho$} & $i$ & $i i$ & $i i i$ & $i v$ \\
\hline Initial mesh with uniform background in Fig.[1(a) & 0.1576 & 0.1029 & 3.6807 & 0.4335 \\
\hline Final mesh with uniform background in Fig.[1(b) & 0.0405 & 0.0274 & 0.2671 & 0.1558 \\
\hline Initial mesh for synthetic phantom in Fig.[2(a) & 0.0858 & 0.0858 & 1.4424 & 0.0858 \\
\hline$k_{D}=0.05$ mesh for synthetic phantom in Fig.[2(c) & 0.0399 & 0.0301 & 0.4789 & 0.1418 \\
\hline$k_{D}=0.30$ mesh for synthetic phantom in Fig.[2(d) & 0.0438 & 0.0251 & 0.6910 & 0.2467 \\
\hline Initial mesh for prostate elastography in Fig.[3(c) & 0.0859 & 0.0856 & 1.4532 & 0.0922 \\
\hline Final mesh for prostate elastography in Fig.[3](d) & 0.0372 & 0.0232 & 0.4668 & 0.1595 \\
\hline
\end{tabular}




\subsection{Modelling a Known Background Distribution}

The methods above assume isotropic meshing of a uniform domain. Anisotropic meshes [3] and effective adjustment of element sizes throughout the mesh [1] have been studied in the literature by incorporating these in the energy definition in (11). However, it is not clear from these and other work, how the information about a feature distribution through the tissue (e.g. distribution of elastic modulus) can be incorporated into the mesh generation, assuming this distribution is known in the continuum a priori. There exist well-established methods, as part of the post-processing stage of FEM simulations, that can refine or modify a mesh based on a computed simulation output such as element strains during deformation. However, this requires the cumbersome process of first running the simulation, which in turn requires a priori knowledge of the boundary conditions such as the fixed and the excited nodes of a mesh during deformation. These boundary conditions may not be known during meshing. Furthermore, their location and nature may change substantially from simulation to simulation such as encountered when a medical tool interacts with different parts of an organ and/or in different directions. Moreover, post-process refinement approaches aim to minimize interpolation error by adjusting node/element density locally. However, such refinement techniques do not formulate an optimum placement for an element intrinsically and refining elements may worsen stiffness matrix conditioning. Also, unlike mechanical engineering, where higher accuracy around high-strain contact areas is desired, medical simulations often require higher accuracy around organ surfaces or anatomical features such as tumors or an overall accuracy in the entire domain. In addition, for a non-uniform property distribution, refining a high-strain element may not always be the right strategy: Consider a 1D example of two elements, one covering a homogeneous soft region and the other one covering an overall stiffer region which internally consists of two disjoint sections with slightly-different elastic properties. Note that, for mesh refinement, subdividing the former element may not necessarily be the optimal strategy despite its higher strain. As a result, we propose the following method based on an error definition that encourages element placement consistent with a given background distribution.

Due to mesh discretization, the FEM models a feature $h(\mathbf{x}), \mathbf{x} \in \tau_{j}$ of the entire space within an element $\tau_{j}$ using a single value $\tilde{h}_{j}$. An error associated with the fitness of this single-value approximation is suggested intuitively to be the $\mathcal{L}_{2}$-norm of the difference between this approximation value and the known background distribution, i.e. $\int_{\tau_{j}}\left(h(\mathbf{x})-\tilde{h}_{j}\right)^{2} d \mathbf{x}$. This is derived below from the elastic strain energy of a linear element, where the known tissue feature is Young's modulus $\mathcal{E}$. For a linear stress-strain relationship, the strain energy of an element can be written in terms of the four corner displacements $\mathbf{u}^{j}$, the constant partial-derivative matrix $B^{j}$ (found from the corner positions), and the material stiffness matrix $C$ as $E_{\text {strain }}^{j}\left(\mathbf{u}^{j}\right)=\frac{1}{2} \int_{\tau^{j}} \mathbf{u}^{j^{T}} B^{j^{T}} C B^{j} \mathbf{u}^{j} d \mathbf{x}$ [4]. In the conventional derivation, $C$ is constant as each element is modeled with a single set of material properties. With $C$ constant, this integration results in the 


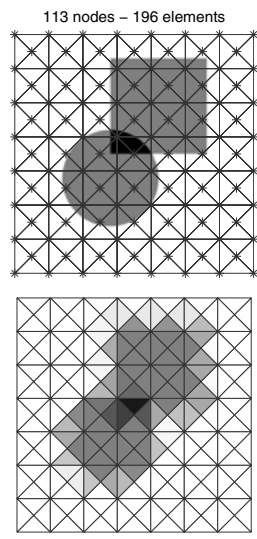

(a)

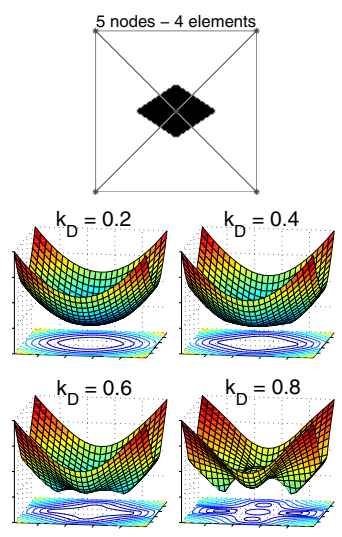

(b)

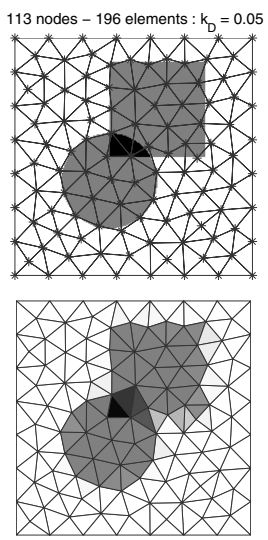

(c)

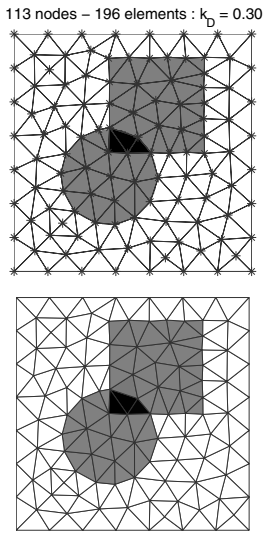

(d)

Fig. 2. An initial synthetic phantom with its discretization (a), the combined error $E$ as a function of center node position for a simple 4-element mesh (b), and the two optimized versions of the initial phantom mesh using $k_{D}=0.05$ (c) and $k_{D}=0.3$ (d)

element volume $v^{j}$. However, $C$ is indeed a linear function of Young's modulus $\mathcal{E}$, the distribution of which is assumed to be known in this paper (i.e., $C=\mathcal{E}(\mathbf{x}) C^{\prime}$ ). Then, the strain energy can be written for a non-uniform element as:

$$
E_{\text {strain }}^{j}\left(\mathbf{u}^{j}\right)=\frac{1}{2} \mathbf{u}^{j^{T}} B^{j^{T}} C^{\prime} B^{j} \mathbf{u}^{j} \int_{\tau^{j}} \mathcal{E}(\mathbf{x}) d \mathbf{x}=\frac{1}{2} \mathbf{u}^{j^{T}} B^{j^{T}} C^{\prime} B^{j} \mathbf{u}^{j} \tilde{\mathcal{E}}_{j} v^{j}
$$

which is satisfied when $\tilde{\mathcal{E}}_{j}$ is the mean of the distribution within the element.

The above observations show that a single-value equivalent of a known background distribution within an element is its mean value. This is presented in Fig.2(a) with a uniform mesh overlaid on a synthetic phantom, where the element colors represent the mean value of the underlying image pixels.

In this paper, an $\mathcal{L}_{2}$-norm discretization error measure $E_{D}$ for a single-value approximation $\tilde{h}_{j}$ and the actual known distribution $h(\mathbf{x})$ is defined as:

$$
E_{D}=\sum_{j} \int_{\tau_{j}}\left|h(\mathbf{x})-\tilde{h}_{j}\right|^{2} d \mathbf{x}=\sum_{j} v^{j} \operatorname{Var}\left(h(\mathbf{x}): \mathbf{x} \in \tau_{j}\right)
$$

where Var is the second-moment of a distribution around its mean. Then, the two error measures $E_{G}$ and $E_{D}$ above are combined into a single error definition in order to derive a variational scheme trading off between element geometry and image representation as:

$$
E=\left(1-k_{D}\right) E_{G}+k_{D} E_{D}
$$

where $k_{D} \in[0,1)$ is a weighting factor. This energy is plotted in Fig.2(b) as a function of the center node position, which defines a unique four-triangle mesh 
over the simple image presented. Note the change of priority from better elementgeometry to lower element-variance as $k_{D}$ increases.

A good approximate initial configuration for geometry error $E_{G}$ can easily be defined as a uniform mesh of regular elements throughout the domain, although it is difficult to define a good initial approximation for $E_{D}$. Therefore, an initial uniform mesh is chosen to start the optimization process. Note that the combined error $E$ depends on how the given image is partitioned by the current tessellation and hence it does not have a simple closed-form expression. In order to minimize this error, a gradient descent search with numerical derivatives has been implemented, where a given node $\mathbf{x}_{i}$ is relocated at each iteration using the error $E_{\Omega_{i}}$ in its 1-ring neighbourhood $\Omega_{i}$, calculated for perturbations in each coordinate axis by a given step length. Sub-step length updates are further achieved using a parabola fit in each individual axis. In our implementation, the step length is defined as a ratio of the distance to the closest element centroid.

\section{Results}

The initial 2D mesh of the synthetic phantom in Fig.2(a) was optimized using two different energy weighting factors yielding the meshes and their discretizations shown in Fig. $2(\mathrm{c}-\mathrm{d})$. The $\rho$-error definitions for this initial mesh and its two different optimized configurations are presented in Table1. Note that all measures, except for the worst element error $(i v)$, were improved, meaning that even for a higher $k_{D}$ value the mesh geometry overall is not worsened while modelling a background image.

The feasibility of our method was next studied on MR images of the brain. An MR slice is first discretized using an initial regular mesh with 128 nodes and 224 triangles as seen in Fig.33(a). This mesh is next optimized for $k_{D}=0.2$ resulting in the discretization in Fig.3(b) in 20 iterations. Although the mesh still remains coarse, a substantial improvement in the representation of the given image is observed once a mesh is optimized using the technique presented. The mesh optimization method was also applied on prostate elastography images. Several methods of tissue parameter identification from displacement estimations have been proposed in the literature [5]. For the purpose of this paper, a simple approximation to the tissue elastic modulus from a tissue strain map $e(x)$ was derived as $1 / e(x)[6]$. An initial 2D mesh with 113 nodes and 196 triangles in Fig. 3(c) takes the form seen in Fig. 3. d) after an optimization taking 15 iterations using $k_{D}=0.3$. A preliminary result of this mesh optimization in $3 \mathrm{D}$ is presented in Fig.3(e-f), where 1078 nodes and 3900 tetrahedra were employed.

The geometric quality of elements, which is traded off in this work for a better discretization of an image, is a major contributor to stiffness matrix conditioning. In this paper, the conditioning $k$ of the stiffness matrices compiled using the initial and final meshes presented have also been studied in order to show that it does not increase significantly, which would negatively affect a simulation. The maximum eigenvalue $\lambda$ of such matrices was proposed in [2] as a good estimate to 


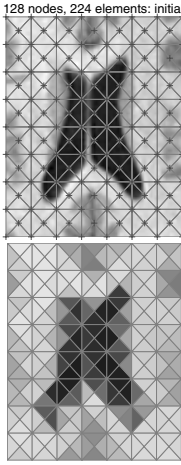

(a)

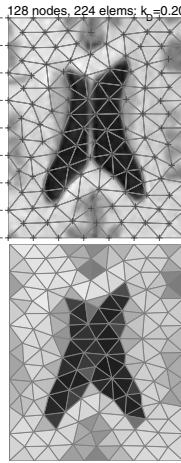

(b)

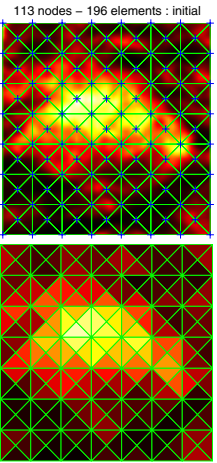

(c)

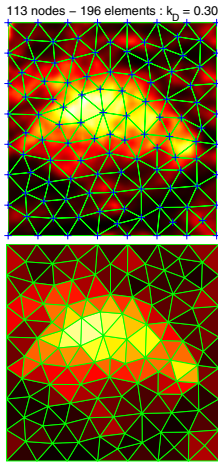

(d)

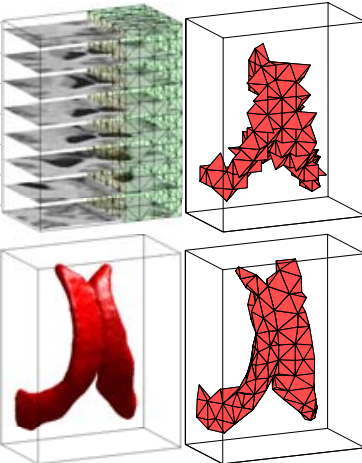

(e)

(f)

Fig. 3. Initial and optimized meshes of ventricles in brain MR images (a-b) and sagittal approximate-stiffness images from prostate elastography (c-d). Slices from a 3D brain MR dataset are shown embedded in an initial 3D mesh (only, part of the mesh is plotted) and the ventricle surface extracted from this volume is presented for comparison purposes (e). Initial and optimized 3D meshes of this MR volume (f). For better 3D visualization, only the largest connected-set of tetrahedra $j$ with $\tilde{h}_{j}$ lower than a chosen threshold are displayed.

the conditioning assuming that the volume of the minimum element is not much smaller than the other elements', which is the case for our nearly uniform-size meshes. The increase in $\lambda$ and $k$ after optimization were $16 \%$ and $7 \%$, respectively. These expected increases were observed to be within acceptable range so as not to introduce numerical instabilities to an FEM application. Furthermore, it was desired to see whether the aggregate strain energy $E_{\text {strain }}^{\mathcal{T}}$ would decrease for a tessellation $\mathcal{T}$ that is optimized as a better discretization of an image using our method. Note that the discretized energy $E_{\text {strain }}^{\mathcal{T}}$ is an overlaid approximant to the exact continuous strain energy and hence bounded below. To compute $k$ and $E_{\text {strain }}$, the top of the meshes were fixed while the bottom was compressed using a plane-stress model. $E_{\text {strain }}$ was observed to be reduced by $5 \%$ for the given constraints after the mesh optimization for the prostate. This agrees with our expectation of improving the strain energy approximation by better aligning the elements with a given parameter distribution.

\section{Discussion}

Some active-contours segmentation approaches that move a parametric surface minimizing the variance inside and outside this surface [7] use a variance-based energy definition similar to our method. However, in contrast to such techniques, where a surface can be evolved at any of its points, linear FEM element faces cannot be modified arbitrarily but instead they are expressed through a connectivity of the mesh nodes. Therefore, an explicit relation between given FEM 
node relocations and the resulting variance change within elements does not exist. Furthermore, there are no longer just two regions, namely inside and outside, but instead multiple disjoint but neighbouring simplex-shaped regions, the FEM elements. Consequently, image feature resolution that a mesh can resolve now depends on the FEM element size. Only uniform meshes were implemented in this paper. Nonetheless, the definition of $E_{G}$ can easily be modified to incorporate a desired local mesh-size information [1] to produce smaller elements that model small anatomical features or higher strain regions, where needed.

Note that the conditioning and strain energy measures presented do not depend only on the mesh and its parametrization, but also are tightly bound by the boundary conditions applied to the FEM. Meshes are optimized by our method assuming that there is no a priori knowledge of the boundary constraints and hence this boundary information is not incorporated into our variational approach. Nevertheless, considering both measures above, an optimized mesh is still expected to rank well for a range of different boundary conditions, one of which was presented in this paper by simply constraining the top and the bottom surface for the prostate mesh. This choice was to model the pubic bone and the ultrasound probe motion during a trans-rectal prostate biopsy.

Although a re-tessellation of nodes ensures a decrease in $E_{G}$, it may possibly increase $E_{D}$ and hence the total energy since it changes the nature of the problem for $E_{D}$. Nonetheless, allowing for re-tessellation is essential to yield geometrically good elements, therefore, re-tessellations are enabled in our optimization scheme during the first $n_{t}$ number of iterations, after which the element connectivity is fixed so the combined energy can be minimized consistently. Alternatively, a tessellation can be accepted only if it decreases the combined energy. In this paper, the initial nodes distributed regularly on the domain boundary are kept fixed during the optimization process and only the position of the internal nodes are optimized. This constraint can be relaxed in various ways to yield better meshes, such as letting these nodes move tangential to the boundary [1]. Note that the errors $E_{G}$ and $E_{D}$ may be in different scales given the image, mesh, and domain dimensions. For similar results among different optimizations using a given $k_{D}$, these error values were normalized to their values in the initial mesh.

\section{Conclusions}

In this paper, an error definition based on FEM interpolation error is combined with a proposed image-representation error and the combined error is optimized to produce high-quality FEM elements that also discretize a given image successfully. With the emerging fields of elastography imaging and tissue parameter identification, this method becomes essential for optimal meshes conforming to such parameters. Note that such an optimized discretization can further be used for a fast approximate segmentation since optimized elements represent an image using far fewer degrees-of-freedom than the underlying pixels. In future work, other optimization techniques will be studied in order to improve on the current descent-based approach. The methods presented do not rely on any assumptions of $2 \mathrm{D}$ and they extend to $3 \mathrm{D}$ easily, as shown by our ventricle-meshing example. 


\section{References}

1. Alliez, P., Cohen-Steiner, D., Yvinec, M., Desbrun, M.: Variational tetrahedral meshing. ACM Trans. on Graphics (procs. of SIGGRAPH) 24(3), 617-625 (2005)

2. Shewchuk, J.R.: What is a good linear element? interpolation, conditioning, and quality measures. In: Eleventh International Meshing Roundtable (2002)

3. Chen, L., Xu, J.: Optimal Delaunay triangulation. Journal of Computational Mathematics 22(2), 299-308 (2004)

4. Zienkiewicz, O.C., Taylor, R.L.: The Finite Element Method. ButterworthHeinemann (2000)

5. Greenleaf, J.F., Fatemi, M., Insana, M.: Selected Methods for Imaging Elastic Properties of Biological Tissues. Annual Review of Biomed. Eng. 5, 57-78 (2000)

6. Ophir, J., Céspedes, I., Ponnekanti, H., Yazdi, Y., Li, X.: Elastography: a method for imaging the elasticity of biological tissues. Ultrasonic Img. 13(2), 111-134 (1991)

7. Chan, T.F., Vese, L.A.: Active contours without edges. IEEE Trans. Image Processing 10(2), 266-277 (2001) 